\title{
DIRICHLETSCHE UND NEUMANNSCHE RANDWERTAUFGABEN IN DER STATISCHEN ELASTIZITÄTSTHEORIE ${ }^{1}$ )
}

\author{
PEKKA NEITTAANMÄKI
}

\section{Einleitung}

Wir betrachten in einem $n$-dimensionalen euklidischen Raum $\boldsymbol{R}^{n}(n \geqq 2)$ einen elastischen. Körper, der im nicht deformierten Zustand das Gebiet $G$ einnehme. Elastische Verformung unter dem Einfluß von Kräften werde im kartesischen Koordinatensystem durch das Vektorfeld $U=\left(U_{1}, \ldots, U_{n}\right)$ der Verschiebungen beschrieben.

Wir legen die Annahmen der linearen Elastizitätstheorie zugrunde: Die Verzerrungen $e_{i k}$ seien durch

$$
e_{i k}(U):=2^{-1}\left(D_{i} U_{k}+D_{k} U_{i}\right), \quad i, k=1, \ldots, n
$$

$\left(D_{i} U_{k}=\partial U_{k} / \partial x_{i}\right)$ gegeben und die durch sie verursachten Spannungen $s_{i k}$ durch

$$
s_{i k}=\sum_{i, m=1}^{n} c_{i k l m} e_{l m}
$$

(das verallgemeinerte Hookesche Gesetz). Die reellwertige Funktionen $c_{i k l m}$ charakterizieren die elastischen Eigenschaften des betrachteten Mediums. Sie genügen den folgenden Symmetrierelationen

$$
c_{i k l m}=c_{l m i k}=c_{m l i k}=c_{i k m l} .
$$

Weiterhin gibt es aus physikalischen Gründen eine positive Konstante $C_{0}$ derart, daß für alle $v_{i k} \in \mathscr{R}:=\left\{v_{i k} \mid v_{i k}=v_{k i}\right\}$,

$$
\sum_{i, j, k, l=1}^{n} c_{i k l m} v_{i k} v_{l m} \geqq C_{0} \sum_{i, l=1}^{n}\left|v_{i l}\right|^{2}
$$

gilt.

Sind die Funktionen $c_{i k l m}$ sonst keinen Einschränkungen unterworfen, so nennt man das Medium inhomogen und anisotrop. Wegen verschiedener Typen elastischer

1) Diese Arbeit ist mit Unterstützung des von der Deutschen Forschungsgemeinschaft getragenen Sonderforschungsbereiches $\mathbf{7 2}$ an der Universität Bonn entstanden. 
Medien (isotroper Fall, rhombischer Fall, kubischer Fall usw.) sei auf Sommerfeld [9] verwiesen.

Falls $F=\left(F_{1}, \ldots, F_{n}\right)$ den Vektor der Volumenkräfte bezeichnet, so genügen die Spannungen den bekannten Gleichgewichtsbedingungen

$$
\sum_{k=1}^{n} D_{k} s_{i k}+F_{i}=0, \quad i=1, \ldots, n .
$$

Indem man die Spannungen mit Hilfe der Beziehungen (1.2) eliminiert, erhält man Bedingungen für das Gleichgewicht in den Verschiebungen; sie lassen sich in Gestalt einer Vektorgleichung aufschreiben

$$
A U:=-\sum_{i, k, l, m=1}^{n} D_{i}\left(c_{i k l m} e_{l m}\right) x_{k}^{0}=F,
$$

wobei $x_{k}^{0}$ den Einheitsvektor in Richtung der Achse $x_{k}$ bedeutet.

Die grundlegenden Randwertprobleme der Elastizitätstheorie sind durch folgende Randbedingungen charakterisiert, wobei wir Homogenität voraussetzen. Hierbei sei mit $\partial G$ der Rand des Gebietes $G$ bezeichnet.

1. Problem bei vorgegebener Randverschiebung (erste Aufgabe):

$$
\left.U\right|_{\partial G}=0 .
$$

2. Problem bei vorgebener Randspannungen (zweite Aufgabe). Der auf $\partial G$ einwirkende Spannungsdichtevektor $t=\sum_{i, k=1}^{n} s_{i k} v_{k} x_{i}^{0}$, wobei $\quad v:=\left(v_{1}, \ldots, v_{n}\right)$ die äußere Normale von $\partial G$ ist, verschwindet:

$$
\left.t\right|_{\partial G}=0 .
$$

Eine Theorie dieser Randwertaufgaben für beschränkte und für Außengebiete (d. h. Gebiete, deren Komplemente kompakt sind) mit glatten Rändern und für homogene isotrope Medien haben Kupradze [4] und Maul [6] mit Integralgleichungsmethoden gegeben. Will man den allgemeinen inhomogenen und anisotropen Fall bei nicht glatten Rändern angehen, dann bieten sich Hilbertraummethoden an.

Die Randwertaufgaben 1 und 2 wurden von vielen Autoren mit dieser Methode für beschränkte Gebiete behandelt. Dazu vergleiche man die Literaturverzeichnisse z. B. in Duvaut-Lions [1], Fichera [2] und Mikhlin [7].

Im zeitharmonischen Fall haben Wacker [10] mit Integralgleichungsmethoden, Weck [11] und Leis [4] mit Hilbertraummethoden entsprechende Randwertprobleme für Außengebiete behandelt.

In dieser Arbeit werden im Falle unbeschränkter elastischer Körper und inhomogener anisotroper Medien Randwertprobleme 1 und 2 mit Hilbertraummethoden untersucht. 


\section{Problemstellung}

Es sei $G \subset \boldsymbol{R}^{n}$ eine offene Menge. Es sei $H^{0}(G)=L^{2}(G)$ der Hilbertraum der reellwertigen im Lebesgueschen Sinne in $G$ meßbaren und quadratintegrierbaren Funktionen bzw. Felder mit dem Skalarprodukt

$$
(U, V)_{0, G}:=\int_{G} U(x) \cdot V(x) d x:=\sum_{i=1}^{n} \int_{G} U_{i}(x) \cdot V_{i}(x) d x
$$

und mit der Norm $\|U\|_{0, G}:=(U, U)_{0, G}^{1 / 2}$.

In der Menge $C^{1}(G)$ der einmal stetig differenzierbaren Funktionen bzw. Felder seien

$$
|U|_{1, G}^{2}:=\sum_{i=1}^{n}\left(D U_{i}, D U_{i}\right)_{0, G}
$$

$\left(D:=\left(D_{1}, \ldots, D_{n}\right)\right)$ und $\|U\|_{1, G}^{2}:=\|U\|_{0, G}^{2}+|U|_{1, G}^{2}$. Es sei

$$
C_{*}^{1}(G):=\left\{U \in C^{1}(G) \mid\|U\|_{1, G}<\infty\right\} .
$$

Für $U, V \in C_{*}^{\mathbf{1}}(G)$ erklärt man das Skalarprodukt

$$
(U, V)_{1, G}:=(U, V)_{0, G}+\sum_{i=1}^{n}\left(D U_{i}, D V_{i}\right)_{0, G} .
$$

Den Hilbertraum $H^{1}(G)$ erhält man durch Vervollständigung der Menge $C_{*}^{\mathbf{1}}(G)$ in Bezug auf $(\cdot, \cdot)_{1, G}$. Die Abschließung der Menge $C_{0}^{\infty}(G)$ der Testfunktionen bzw. Testfelder von $G$ im $\operatorname{Raum~} H^{1}(G)$ sei $H_{0}^{1}(G)$.

Wir definieren mit den Gewichten (von der Dimension $n$ des Raumes $\boldsymbol{R}^{n}$ abhängend)

$$
g(x):=\left\{\begin{array}{lll}
1 /((1+|x|) \ln (e+|x|)) & \text { für } & n=2 \\
1 /(1+|x|) & \text { für } & n \geqq 3
\end{array}\right.
$$

die gewichteten $L^{2}$-Räume

und

$$
L_{g}^{2}(G):=\left\{U \mid g U \in L^{2}(G)\right\}
$$

mit den Skalarprodukten

$$
L_{\mathbf{1} / g}^{2}(G):=\left\{U \mid g^{-1} U \in L^{2}(G)\right\}
$$

$$
\langle U, V\rangle_{0, g, G}:=\int_{G} g^{2} U \cdot V d x, \quad U, V \in L_{g}^{2}(G)
$$

bzw.

$$
\langle U, V\rangle_{0,1 / g, G}:=\int_{G} g^{-2} U \cdot V d x, \quad U, V \in L_{1 / g}^{2}(G) .
$$

Für Felder $U \in C^{1}(G)$ können wir den Ausdruck

$$
\|U\|_{1, g, G}^{2}:=\int_{G} g^{2} U \cdot U d x+\sum_{i=1}^{n} \int_{G} D U_{i} \cdot D U_{i} d x
$$


bilden. Es sei

$$
C_{g^{*}}^{1}(G):=\left\{U \in C^{1}(G) \mid\|U\|_{1, g, G}<\infty\right\} .
$$

Für Felder $U, V \in C_{g_{*}}^{\mathbf{1}}(G)$ erklärt man das Skalarprodukt

$$
\langle U, V\rangle_{1, g, G}:=\int_{G} g^{2} U \cdot V d x+\sum_{i=1}^{n} \int_{G} D U_{i} \cdot D V_{i} d x
$$

Der Raum $H_{g}^{1}(G)$ wird als Vervolltändigung von $C_{g *}^{1}(G)$ in Bezug auf $\langle\cdot, \cdot\rangle_{1, g, G}$ definiert. Die Abschließung von $C_{0}^{\infty}(G)$ im Raum $H_{g}^{1}(G)$ wird mit $H_{g 0}^{1}(G)$ bezeichnet.

Zur Formulierung von Randwertaufgaben bilden wir nun die Bilinearform

$$
B(U, V):=\sum_{i, k, l, m=1}^{n} \int_{G} c_{i k l m} e_{l m}(U) e_{i k}(V) d x .
$$

Im folgenden nehmen wir an:

Voraussetzung 2.1. Die Koeffizienten $c_{i k l m}$ des Operators $A$ sind reellwertige, beschränkte und meßbare Funktionen auf $\bar{G}$ und genügen den Relationen (1.3) und (1.4). stellen:

Jetzt können wir unsere Randwertaufgaben für unbeschränkte Gebiete $G$

Problem 1 (Die erste (Dirichletsche) Randwertaufgabe). In $G \subset \boldsymbol{R}^{n}$ sei $F \in L_{\mathbf{1} / \mathbf{g}}^{2}(G)$ gegeben. Es wird die Gesamtheit der Funktionen $U \in H_{g 0}^{1}(G)$ (der schwachen Lösungen des Dirichletschen Problems) gesucht die der Gleichung

$$
B(U, V)=(F, V)_{0, G} \text { für alle } V \in H_{g 0}^{1}(G)
$$

genügen.

Problem 2 (Die zweite (Neumannsche) Randwertaufgabe). In $G \subset \boldsymbol{R}^{n}$ sei $F \in L_{1 / g}^{2}(G)$ gegeben. Es wird die Gesamtheit der Funktion $U \in H_{g}^{1}(G)$ (der schwachen Lösungen des Neumannschen Problems) gesucht die der Gleichung (1.10) für alle $V \in H_{g}^{1}(G)$ genügen.

Wir werden zeigen, daß Problem 1 für beliebiges $G$ eindeutig lösbar ist (Satz 4.1). Wenn $G$ ein Außengebiet mit Kegeleigenschaft ist (siehe Fichera [2], S. 382), können wir zeigen, daß Problem 2 für $n \geqq 3$ eine eindeutige Lösung hat und daß im Falle $n=2$ die Fredholmsche Alternative gilt (Satz 4.2).

\section{Erste und zweite Kornsche Ungleichung in gewichteten Sobolevräumen}

Bei den Randwertaufgaben der Elastizitätstheorie 'spielen die Kornschen Ungleichungen im Beweis der strengen Koerzitivität bzw. der Koerzitivität der Bilinearform eine ähnliche Rolle wie die Poincaréschen Ungleichungen bei den Randwertaufgaben der Potentialtheorie: Nach (1.4) und (1.1) gilt für alle $U \in H^{1}(G)$

$$
B(U, U) \geqq C_{0} \sum_{i, k=1}^{n}\left\|D_{i} U_{k}+D_{k} U_{i}\right\|_{0, G}^{2} .
$$


Im Falle beschränkter Gebiete kann man mit Hilfe der Kornschen Ungleichungen die rechte Seite von (3.1) weiter abschätzen. Die zweite Kornsche Ungleichung lautet (vgl. Fichera [2], S. 382): Zu einem beschränkten Gebiet $G$ mit Kegeleigenschaft gibt es eine positive Konstante $C$ derart, daß

$$
\sum_{i, k=1}^{n}\left\|D_{i} U_{k}+D_{k} U_{i}\right\|_{0, G}^{2} \geqq C\|U\|_{1, G}^{2}-\|U\|_{0, G}^{2}
$$

für alle $U \in H^{1}(G)$ gilt.

Leis hat in [5] gezeigt, daß (3.2) auch für Außengebiete mit Kegeleigenschaft gilt. In der statischen Elastizitätstheorie im Falle eines unbeschränkten Körpers ist diese Ungleichung nicht relevant, weil man nicht erwarten kann, daß die Lösungen quadratintegrierbar sind. Deswegen werden wir zuerst zeigen, daß mit zwei positiven Konstanten $C_{1}$ und $C_{2}$ für alle $U \in H_{\mathrm{g}}^{1}(G)$

$$
\sum_{i, k=1}^{n}\left\|D_{i} U_{k}+D_{k} U_{i}\right\|_{0, G}^{2} \geqq C_{1}|U|_{1, G}^{2}-C_{2}\|U\|_{0, G(R)}^{2}
$$

gilt, wobei $G(R):=\left\{x \in G|| x \mid=\left(x_{1}^{2}+\ldots+x_{n}^{2}\right)^{1 / 2}<R\right\} \subset G$ ist. Dazu setzen wir woraus, daß $G$ ein Außengebiet ist und daß für $G(R)$ Ungleichung (3.2) gültig ist.

Im Falle der Dirichletsche Randwertaufgabe gilt Ungleichung (3.3) mit $C_{2}=0$ und zwar für beliebige Gebiete: Durch partielle Integration erhalten wir nämlich für alle $\Phi=\left(\Phi_{1}, \ldots, \Phi_{n}\right) \in C_{0}^{\infty}(G)$

$$
\begin{gathered}
\sum_{i, k=1}^{n}\left\|D_{i} \Phi_{k}+D_{k} \Phi_{i}\right\|_{0, G}^{2} \\
=2 \sum_{i, k=1}^{n}\left\{\left\|D_{i} \Phi_{k}\right\|_{0, G}^{2}+\int_{G}\left(D_{i} \Phi_{k}\right)\left(D_{k} \Phi_{i}\right) d x\right\} \\
=2 \sum_{i, k=1}^{n}\left\|D_{i} \Phi_{k}\right\|_{0, G}^{2}+2\|\operatorname{div} \Phi\|_{0, G}^{2} \\
\geqq 2|\Phi|_{1, G}^{2} .
\end{gathered}
$$

Zum Beweis von Ungleichung (3.3) sei $R>0$ so groß, daß $R^{n}-\bar{G} \subset K(R):=$ $\left\{x \in \boldsymbol{R}^{n}|| x \mid<R\right\}$ gilt. Sei $\varphi \in C_{0}^{\infty}([0,3 R))$ eine nichtwachsende Glättungsfunktion mit

Sei

$$
\varphi(|x|)=\left\{\begin{array}{lll}
1 & \text { für } & |x| \leqq R \\
1 / 2 & \text { für } & |x|=2 R \\
0 & \text { für } & |x| \geqq 3 R .
\end{array}\right.
$$

$$
L(U, V):=\sum_{i, k=1}^{n}\left(D_{i} U_{k}+D_{k} U_{i}, D_{i} V_{k}+D_{k} V_{i}\right)_{0, G} .
$$

Dann gilt mit $\eta \in C_{0}^{\infty}\left(\boldsymbol{R}^{n}\right)$

$$
L(\eta, \eta)=L(\varphi \eta, \varphi \eta)+2 L(\varphi \eta,(1-\varphi) \eta)+L((1-\varphi) \eta,(1-\varphi) \eta) .
$$


Weil der Träger $\operatorname{Trg} \varphi \eta$ von $\varphi \eta$ in $K(3 R)$ enthalten ist, erhalten wir nach (3.2) mittels (3.5) mit allgemeinen, von $\varphi$ und $\eta$ unabhängigen, positiven Konstanten $C_{i}$

$$
\begin{gathered}
L(\varphi \eta, \varphi \eta) \geqq C_{1}\|\varphi \eta\|_{1, G(3 R)}^{2}-\|\varphi \eta\|_{0, G(3 R)}^{2} \\
\geqq C_{1} 4^{-1}\left(1-\varepsilon_{1}\right)|\eta|_{1, G(2 R)}^{2}-C_{2} \varepsilon_{1}^{-1}\|\eta\|_{0, G(3 R)}^{2},
\end{gathered}
$$

wobei $G(R):=G \cap K(R)$ und $0<\varepsilon_{1}<1$ ist.

Weil $(1-\varphi) \eta \in C_{0}^{\infty}(B(R))(B(R):=\{x \in G|| x \mid>R\})$ ist, folgt entsprechend aus (3.4) mittels (3.5)

$$
\begin{gathered}
L((1-\varphi) \eta,(1-\varphi) \eta) \geqq 2|(1-\varphi) \eta|_{1, B(R)}^{2} \\
\geqq 2|\eta|_{1, B(3 R)}^{2}+4^{-1}\left(2-\varepsilon_{2}\right)|\eta|_{1, K(2 R, 3 R)}^{2}-C_{3} \varepsilon_{2}^{-1}\|\eta\|_{0, K(R, 3 R)}^{2},
\end{gathered}
$$

wobei $K\left(R_{1}, R_{2}\right):=\left\{x \in G\left|R_{1}<\right| x \mid<R_{2}\right\}$ und $0<\varepsilon_{2}<2$ ist.

Für den letzten Term in Gleichung (3.6) erhalten wir nach partieller Integration wie oben mit $\varepsilon_{3}>0$

$$
\begin{gathered}
L(\varphi \eta,(1-\varphi) \eta) \\
\geqq \sum_{i, k=1}^{n} \int_{G}(1-\varphi) \varphi\left|D_{i} \eta_{k}+D_{k} \eta_{i}\right|^{2} d x-\varepsilon_{3}|\eta|_{1, K(R, 3 R)}^{2}-C_{4} \varepsilon_{3}^{-1}\|\eta\|_{0, G(3 R)}^{2} \\
\geqq-\varepsilon_{3}|\eta|_{1, K(R, 3 R)}^{2}-C_{4} \varepsilon_{3}^{-1}\|\eta\|_{0, G(3 R)}^{2} .
\end{gathered}
$$

Die Behauptung (3.3) folgt jetzt durch (3.6) nach geeigneter Wahl von $\varepsilon_{1}, \varepsilon_{2}$ und $\varepsilon_{3}$ aus den Ungleichungen (3.7)-(3.9).

Um die gewünschte strenge Koerzitivität bzw. Koerzitivität von $B$ zu erhalten, brauchen wir noch folgende Variante der Poincaréschen Ungleichung für Vektorfelder von $H_{g 0}^{1}(G)$ bzw. von $H_{g}^{1}(G)$ :

Lemma 3.1. Es sei $G \subset \boldsymbol{R}^{n}$ ein beliebiges Gebiet. Dann gibt es eine von $U$ unabhängige Konstante $C_{1}>0$ derart, daß

$$
\|U\|_{1, g, G} \leqq C_{1}|U|_{1, G}
$$

für alle $U \in H_{g 0}^{1}(G)$ gilt.

Es sei $G \subset \boldsymbol{R}^{n}$ ein Außengebiet mit Kegelbedingung (sogar mit Segmenteigenschaft). Dann gibt es eine von $U$ unabhängige Konstante $C_{2}>0$ derart, daß

$$
\|\mid U\|_{1, g, G} \leqq C_{2}\left(|U|_{1, G}+\|U\|_{0, K(2 R, 3 R)}\right)
$$

für alle $U \in H_{g}^{1}(G)$ gilt, wobei die Konstante $R$ so groß ist, daß $R^{n}-\overline{K(R)} \subset G$ gilt.

Beweis. A. Ohne Einschränkung kann man $K(e) \subset \boldsymbol{R}^{n}-\bar{G}$ annehmen (vgl. [8], S. 30-31. Wir erklären in $G$ die Gewichtsfunktionen $g^{\prime}$ :

$$
g^{\prime}(x):=\left\{\begin{array}{lll}
1 /(|x| \ln |x|) & \text { für } & n=2 \\
1 /|x| & \text { für } & n \geqq 3
\end{array}\right.
$$

und beweisen für $U_{i} \in C_{0}^{\infty}(G)(i=1, \ldots, n)$ die Ungleichung

$$
\left\|g^{\prime} U_{i}\right\|_{0, G} \leqq 2\left|U_{i}\right|_{1, G} .
$$


Für $U_{i} \in C_{0}^{\infty}(G)$ erklären wir einen positiven Ausdruck

$$
I_{\sigma(n)}\left(U_{i}\right):=\left\|D U_{i}+\sigma(n) g^{\prime} \hat{x} U_{i}\right\|_{0, G}^{2}
$$

mit $\hat{x}=x /|x|, \sigma(n)=-1$ für $n=2$ und $\sigma(n)=1$ für $n \geqq 3$. Durch partielle Integration $\epsilon$ rhalten wir

$$
I_{\sigma(n)}\left(U_{i}\right) \leqq\left|U_{i}\right|_{1, G}^{2}
$$

Aus (3.13) ergibt sich

$$
\begin{aligned}
\left\|g^{\prime} U_{i}\right\|_{0, G}^{2} & \leqq \\
& \leqq 4 U_{i}+\sigma(n) g^{\prime} U_{i}^{2}-D U_{i} \|_{0, G}^{2} .
\end{aligned}
$$

Wegen der Äquivalenz der Gewichtsfunktionen $g$ und $g^{\prime}$ erhalten wir aus (3.12) Behauptung (3.10).

B. Zum Beweis von (3.11) betrachten wir eine Glättungsfunktion $\varphi \in C_{0}^{\infty}(K(3 R))$ mit $\left.\varphi\right|_{K(2 R)} \equiv 1$. Für $U_{i} \in H_{g}^{1}(G)(i=1, \ldots, n)$ erhalten wir wegen $\operatorname{Trg} \varphi U_{i} \subset K(3 R)$ und $\operatorname{Trg}(1-\varphi) U_{i} \subset B(R)$ mit Hilfe der Poincaréschen Ungleichung und (3.10) (vgl. [8], Beweis von Satz II. 3.3)

$$
\begin{aligned}
& \left\|U_{i}\right\|_{1, g, G} \leqq\left\|\varphi U_{i}\right\|_{1, g, G}+\left\|(1-\varphi) U_{i}\right\|_{1, g, G} \\
& \leqq C_{1}\left(\left|U_{i}\right|_{1, G(3 R)}+\left\|U_{i}\right\|_{0, K(2 R, 3 R)}\right) \\
& +C_{2}\left(\left|U_{i}\right|_{1, B(3 R)}+\left\|U_{i}\right\|_{0, K(2 R, 3 R)}\right),
\end{aligned}
$$

wobei $C_{1}>0$ und $C_{2}>0$ unabhängig von $U_{i}$ sind. Behauptung (3.11) folgt dann unmittelbar aus (3.14).

Aus (3.4) und (3.10) bzw. (3.3) und (3.11) erhalten wir

Satz 3.2. Die erste Kornsche Ungleichung in gewichteten Sobolevräumen: Es sei $G \subset \boldsymbol{R}^{n}$ ein beliebiges unbeschränktes Gebiet. Dann gibt es eine von U unabhängige Konstante $C_{1}>0$ derart, daß die Ungleichung

$$
\sum_{i, k=1}^{n}\left\|D_{i} U_{k}+D_{k} U_{i}\right\|_{0, G}^{2} \geqq C_{1}\|U\|_{1, g, G}^{2}
$$

für alle $U \in H_{g 0}^{1}(G)$ gilt.

Die zweite Kornsche Ungleichung in gewichteten Sobolevräumen: Es sei $G \subset \boldsymbol{R}^{n}$ ein Außengebiet mit Kegeleigenschaft. Dann gibt es zwei von U unabhängigen Konstanten $C_{2}>0$ und $C_{3}>0$ derart, daß die Ungleichung

$$
\sum_{i, k=1}^{n}\left\|D_{i} U_{k}+D_{k} U_{i}\right\|_{0, G}^{2} \geqq C_{2}\|U\|_{1, g, G}^{2}-C_{3}\|U\|_{0, G(3 R)}^{2}
$$

für alle $U \in H_{g}^{1}(G)$ gilt, wobei $R>0$ mit $R^{n}-\overline{K(R)} \subset G$ ist. 


\section{Lösbarkeitsaussagen für Randwertaufgaben}

Nach Voraussetzung 2.1 und nach Definition der Norm $\||\cdot|\|_{1, g, G}$ ist die Bilinearform $B(U, V)$ in $H_{g 0}^{1}(G)$ bzw. in $H_{g}^{1}(G)$ beschränkt: Es gilt mit einer positiven Konstanten $C_{1}$

$$
B(U, V) \leqq C_{1}\left\|U\left|\left\|_{1, g, G} !\right\| V\right|\right\|_{1, g, G}
$$

für alle $U, V \in H_{g 0}^{1}(G)$ bzw. alle $U, V \in H_{g}^{1}(G)$.

Nach (3.1) und (3.15) ist $B$ in $H_{g 0}^{1}(G)$ streng koerzitiv: Es gibt ejne positive Konstante $C_{2}$ derart, daß

$$
B(U, U) \geqq C_{2}|||U| \|_{1, g, G}^{2}
$$

für alle $U \in H_{g 0}^{1}(G)$ gilt.

Wegen

$$
\left|(F, V)_{0, G}\right| \leqq\left.\left.|||F|\right|_{0,1 / g, G}|||V|\right|_{1, g, G}
$$

ist das Funktional $a(V):=(F, V)_{0, G}$ mit $F \in L_{1 / g}^{1}(G)$ in $H_{g 0}^{1}(G)$ beschränkt. Mittels (4.1) und (4.2) erhalten wir daher aus dem Satz von Lax und Milgram

Satz 4.1. Voraussetzung 2.1 sei erfüllt. Dann ist die Dirichletsche Randwertaufgabe für unbeschränkte Gebiete $G$ und für $F \in L_{1 / g}^{2}(G)$ eindeutig lösbar.

Die Behandlung der zweiten Randwertaufgabe ist schwieriger, weil aus der zweiten Kornschen Ungleichung nur die Koerzitivität der Bilinearform $B$ folgt: Die zweite Aussage von Satz 3.2 angewandt auf Ungleichung (3.1) gibt für alle $U \in H_{g}^{1}(G)$ die Ungleichung

$$
B(U, U) \geqq \mu\|\| U\left\|_{1, g, G}^{2}-\lambda\right\| U \|_{0, G(3 R)}^{2},
$$

wobei $\mu$ und $\lambda$ positive Konstanten sind. Mit Hilfe der Stabilität der Fredholmeigenschaft gegenüber kompakten Störungen erhalten wir aus (4.3)

Satz 4.2. Es sei ein Außengebiet mit Kegeleigenschaft und sei Voraussetzung 2.1 erfüllt. Wenn die Raumdimension $n \geqq 3$ ist, ist die Neumannsche Randwertaufgabe für $F \in L_{1 / g}^{2}(G)$ eindeutig lösbar.

Wenn $n=2$ ist, hat die Neumannsche Randwertaufgabe für $F \in L_{1 / g}^{2}(G)$ genau dann eine Lösung, falls die Bedingung

$$
(F, V)_{0, G}=0
$$

für alle $V \in \mathscr{P}_{0}:=\boldsymbol{R}^{2}$ erfüllt ist. Diese Lösung ist dann bis auf ein beliebiges Element aus $\mathscr{P}_{0}$ eindeutig bestimmt.

Beweis. A. Wir erklären in $H_{g}^{1}(G) \times H_{g}^{1}(G)$ eine Bilinearform

$$
\hat{B}(U, V):=B(U, V)+\lambda\left(\chi_{G(3 R)} U, V\right)_{0, G},
$$

wobei $\chi_{G(3 R)}$ die charakteristische Funktion von $G(3 R)$ ist, und führen einen Operator $\hat{A}$ durch diese Form ein. 
Es sei $D(\hat{A})\left(\subset L_{g}^{2}(G)\right)$ die Menge derjenigen Elemente $U \in H_{g}^{1}(G)$, zu denen je ein $F \in L_{1 / g}^{2}(G)$ mit

$$
B(U, V)=(F, V)_{0, G}
$$

für alle $V \in H_{g}^{1}(G)$ existiert. Durch

$$
\hat{A} U:=F \quad \text { für } \quad U \in D(\hat{A})
$$

wird $\hat{A}: D(\hat{A}) \rightarrow L_{1 / g}^{2}(G)$ erklärt.

Der Operator $\hat{A}$ hat in $L_{g}^{2}(G)$ einen dichten Definitionsbereich (vgl. etwa Weidmann [12], Satz 5.36). Wie in [8], Satz II. 3.12, kann man beweisen, daß der Operator $\hat{A}$ ein Fredholmoperator mit dem Index ind $\hat{A}=0$ ist (vgl. Kato [3], § IV. 5.1).

Ferner können wir wie in [8], §II. 3.5, einen Operator $\Gamma: D(\Gamma) \rightarrow L_{1 / g}^{2}(G)$ mit $D(\Gamma):=D(\hat{A})$ und

$$
\Gamma U:=\lambda \chi_{G(3 R)} U, \text { für } U \in D(\Gamma)
$$

definieren und beweisen, daß $\Gamma$ ein $\hat{A}$-kompakter Operator ist (vgl. Kato [3], $\S$ IV. 1.3).

Mit Hilfe der Stabilitätsergebnisse für Fredholmoperatoren (vgl. Kato [3], $\S$ IV. 5.3) erhalten wir: Der Operator $A:=\hat{A}-\Gamma$ mit $D(A)=D(\hat{A})$ ist ein Fredholmoperator mit dem Index 0.

B. Nach (3.1) jst

$$
N(A):=\left\{U \in H_{g}^{1}(G) \mid B(U, V)=0 \text { für alle } V \in H_{g}^{1}(G)\right\}
$$

in $\mathscr{P}_{1} \cap H_{g}^{1}(G)$ enthalten, wobei $\mathscr{P}_{1}$ durch

$$
\mathscr{P}_{1}:=\left\{U=\left(U_{1}, \ldots, U_{n}\right) \mid U_{i}=a_{i}+\sum_{j=1}^{n} b_{i j} x_{j}, a_{i}, b_{i j} \in \boldsymbol{R} \text { und } b_{i j}=-b_{j i}\right\}
$$

definiert ist.

Weil $B(U, V)=0$ für alle $U \in \mathscr{P}_{1} \cap H_{g}^{1}(G)$ und alle $V \in H_{g}^{1}(G)$ gilt, ist

$$
N(A)=\mathscr{P}_{1} \cap H_{g}^{1}(G) .
$$

Andererseits gilt $\mathscr{P}_{1} \cap H_{g}^{1}(G)=\mathscr{P}_{0}$ für $n=2$ und $\mathscr{P}_{1} \cap H_{g}^{1}(G)=\{0\}$ für $n \geqq 3$. Folglich ist im Falle $n \geqq 3$ auch $N(A)=\{0\}$. Dies zusammen mit der Tatsache ind $A=0$ liefert den ersten Teil von Satz 4.2.

Im Falle $n=2$ erklären wir $Q:=\left\{V=g^{2} U \mid U \in \mathscr{P}_{0}\right\}$. Man kann beweisen, da $\beta$ $Q$ im Nullraum $N\left(A^{*}\right)$ des adjungierten Operators $A^{*}$ enthalten ist. Weil wegen ind $A=0$ die Relation $\operatorname{dim} Q=\operatorname{dim} N(A)=\operatorname{dim} N\left(A^{*}\right)$ gilt, ist $N\left(A^{*}\right)=Q$. Dies zusammen mit der Tatsache $N\left(A^{*}\right)=R(A)^{\perp}$ (orthogonales Komplement der Wertemenge des Operators A, vgl. Kato [3], Theorem 5.13, S. 234) liefert die zweite Aussage von Satz 4.2. 


\title{
Literatur
}

[1] Duvaut, G., und J. L. Lions: Inequalities in mechanics and physics. - Die Grundlehren der mathematischen Wissenschaften 219. Springer-Verlag, Berlin-Heidelberg-New York, 1976.

[2] Fichera, G.: Existence theorems in elasticity. - Handbuch der Physik VI a/2. Festkörpermechanik II. Springer-Verlag, Berlin-Heidelberg-New York, 1972, 347-389.

[3] Kato, T.: Perturbation theory for linear operators. - Die Grundlehren der mathematischen Wissenschaften 132. Springer-Verlag, Berlin-Heidelberg-New York, 1966.

[4] Kupradze, V. D.: Potential methods in the theory of elasticity. - Israel Program for Scientific Translations, Jerusalem, 1965.

[5] LeIs, R.: Zur Theorie elastischer Schwingungen- Berichte der Gesellschaft für Mathematik und Datenverarbeitung Bonn 72, St. Augustin, 1973.

[6] Maul, J.: Zur Lösung von Aufgaben der ebenen Elastostatik in unendlichen Körpern. - Beiträge Anal. 9, 1976, 55-60.

[7] Mikhlin, S. G.: The problem of the minimum of a quadratic functional. - Holden-Day, Inc., San Francisco, 1970.

[8] Neittaanmäki, P.: Randwertaufgaben zur Plattengleichung. - Ann. Acad. Sci. Fenn. Ser. A I Math. Dissertationes 16, 1978, 1-71.

[9] Sommerfeld, A.: Vorlesungen über theoretische Physik. II. Mechanik der deformierbaren Medien. - 6. Auflage, Akademische Verlagsgesellschaft Geest \& Portig K.-G., Leipzig, 1970.

[10] WACKeR, H. M.: Existenz und Eindeutigkeitssätze für die erste und zweite Randwertaufgabe des Außenraumproblems der Gleichung elastischer Schwingungen. - Bonn. Math. Schr. 31, 1968, 1-61.

[11] Weck, N.: Zur mathematischen Theorie stationärer Schwingungen elastischer Medien. Bonn. Math. Schr. 35, 1969, 1-85.

[12] WeidmanN, J.: Lineare Operatoren in Hilberträumen. - Mathematische Leitfäden, B. G. Teuber, Stuttgart, 1976.

\author{
Universität Jyväskylä \\ Mathematisches Institut \\ Sammonkatu 6 \\ SF-40100 Jyväskylä 10 \\ Finnland
}

Eingegangen am 16. August 1979 